\title{
HAAR SERIES
}

\author{
BY \\ JAMES R. MCLAUGHLIN( $\left.{ }^{1}\right)$
}

1. Introduction. The orthonormal system introduced by A. Haar has been studied by P. L. Ul'janov [21]-[28] and numerous other authors. It has been our object to continue these investigations using a classical real variable approach. The main body of this paper deals with Haar series, although there are results on Walsh, trigonometric, and other orthonormal series.

Because of the large number of individual results we will not try to describe them all here but only state one theorem from each section. We will first give some definitions and basic properties of the Haar system.

Definition 1.1. Haar's orthonormal system $\left\{\chi_{m}(t)\right\}$ is defined as follows on $[0,1]: \chi_{0}(t) \equiv 1$ and for $m=2^{n}+k$ with $0 \leqq k<2^{n}, n=0,1, \ldots$

$$
\begin{aligned}
\chi_{m}(t) & =2^{n / 2}, & & t \in\left(k / 2^{n},(k+1 / 2) / 2^{n}\right), \\
& =-2^{n / 2}, & & t \in\left((k+1 / 2) / 2^{n},(k+1) / 2^{n}\right), \\
& =0, & & t \in[0,1] \backslash\left[k / 2^{n},(k+1) / 2^{n}\right],
\end{aligned}
$$

and at the three remaining points we let $\chi_{m}(t)$ be equal to the average of the right and left hand limits.

Definition 1.2. For $f(t) \in L(0,1)$ we call $a_{m}(f)=\int_{0}^{1} f(t) \chi_{m}(t) d t, m=0,1, \ldots$, the Haar-Fourier coefficients of $f$ and $\sum_{m=0}^{\infty} a_{m}(f) \chi_{m}(t)$ the Haar-Fourier series of $f$.

It follows easily [1, p. 49] that for $S_{m}\left(t_{0}\right)=\sum_{i=0}^{m} a_{i}(f) \chi_{i}\left(t_{0}\right), m=2^{n}+k, 0 \leqq k<2^{n}$, $n=0,1, \ldots$, we have

(i) If $t_{0}$ is a dyadic irrational, then

$$
S_{m}\left(t_{0}\right)=\frac{1}{\left|I_{m}\right|} \int_{I_{m}} f(t) d t
$$

where $t_{0} \in I_{m}$, which is an interval of length $2^{-n}$ or $2^{-n-1}$.

(ii) If $t_{0}=p / 2^{q}$ is a dyadic rational, then for $m \geqq 2^{q}$

$$
S_{m}\left(t_{0}\right)=\frac{1}{2\left|I_{m}\right|} \int_{I_{m}} f(t) d t+\frac{1}{2\left|J_{m}\right|} \int_{J_{m}} f(t) d t
$$

where $I_{m}=\left(t_{0}-\alpha, t_{0}\right), J_{m}=\left(t_{0}, t_{0}+\beta\right)$, and where $\alpha=\beta=2^{-n-1}$ or $\alpha=2^{-n-1}$ and $\beta=2^{-n}$.

The above facts lead to the following results of A. Haar [1, p. 47]:

Presented to the Society, January 24, 1967 under the title Absolute convergence of Haar series; received by the editors August 16, 1967 and, in revised form, January 10, 1968.

( $\left.{ }^{1}\right)$ This research was supported by NSF and NASA fellowships and is part of the author's doctoral dissertation written at Wayne State University. The author wishes to express his appreciation to his advisor, Professor Daniel Waterman, for his encouragement and guidance. 
THEOREM A. If $f(t)$ is integrable on $(0,1)$, then the Haar-Fourier series of $f(t)$ converges to $f(t)$ a.e.

THEOREM B. If $f(t)$ is integrable on $(0,1)$, then the Haar-Fourier series of $f(t)$ converges to $f(t)$ at every point of continuity of $f(t)$, and converges uniformly in every interval in which $f(t)$ is uniformly continuous.

In view of equations (1.1) and (1.2) and properties of approximately continuous functions [13, p. 292] we also have the following:

THEOREM 1.1. If $f(t)$ is bounded and approximately continuous on $[0,1]$, then the Haar-Fourier series of $f(t)$ converges to $f(t)$ on $[0,1]$.

THEOREM 1.2. If $t_{0}$ is a dyadic irrational and $f(t)$ is integrable on $(0,1)$ and is bounded and has an approximate limit at $t_{0}$, then the Haar-Fourier series of $f(t)$ converges at $t_{0}$.

THEOREM 1.3. If $t_{0}$ is a dyadic rational and $f(t)$ is integrable on $(0,1)$ and is bounded and has an approximate right and left hand limit at $t_{0}$, then the HaarFourier series of $f(t)$ converges at $t_{0}$.

In Theorem 1.1 the convergence is not uniform in general. In fact, since the Haar functions are continuous at dyadic irrationals and have right and left hand limits everywhere, we have

THEOREM 1.4. If $\sum_{i=0}^{m} a_{i} \chi_{i}(t)$ converges to $f(t)$ uniformly on $[0,1]$, then $f(t)$ is continuous at dyadic irrationals and has right and left hand limits everywhere.

REMARK 1.1. The condition of boundedness in Theorem 1.1 cannot be omitted since approximate continuity at $t_{0}$ does not imply that $S_{m}\left(t_{0}\right)$ converges to $f\left(t_{0}\right)$.

We now give a representative theorem from each of the sections which follow.

In $\$ 2$ we shall study absolute convergence of Haar series and prove that the Haar-Fourier series of a function of bounded variation converges absolutely at all dyadic rationals and that for every dyadic irrational there exists an absolutely continuous function whose Haar-Fourier series diverges absolutely for that number.

In $\S 3$ we investigate relationships between the numbers $a_{m}$ and the function $f(t)$ which represents the sum of the series $\sum a_{m} \chi_{m}(t)$ and prove that if $f(t)$ has the Darboux property and $a_{m}=o\left(m^{-3 / 2}\right)$, then $f$ is a constant.

In $\$ 4$ we will give some applications of Haar series to general orthonormal series and show that if $\left\{\phi_{m}(t)\right\}$ is o.n. on $[a, b]$ and $M_{m}$ denotes the supremum of the $m$ th function, then

(i) $\int_{a}^{b} f(t) \phi_{m}(t) d t=o\left(M_{m}\right)$ for every integrable $f$.

(ii) For unbounded complete orthonormal systems this result may not in general be improved. 
2. Absolute convergence of Haar series. We shall consider here some results concerning the convergence of the series

$$
\sum_{m=0}^{\infty}\left|a_{m}(f) \chi_{m}(t)\right|
$$

and

$$
\sum_{m=0}^{\infty}\left|a_{m}(f)\right|
$$

Z. Ciesielski and J. Musielak proved [4, p. 62, Theorem 1]

THEOREM C. If $\sum_{n=1}^{\infty} \omega\left(f, 2^{-n}\right)<\infty$, where $\omega(f, \delta)$ denotes the modulus of continuity of $f$, then (2.1) converges uniformly on $[0,1]$.

If we assume that $f(t)$ is only continuous on $[0,1]$, then the conclusion in Theorem $\mathrm{C}$ is not true. In fact A. M. Olevskir has shown [14, p. 1382] that for every complete orthonormal system there is a continuous function whose Fourier series is absolutely divergent a.e.

The following problem was posed by Ciesielski and Musielak [4, p. 65]: Does the convergence of (2.2) imply the uniform convergence of (2.1), $a_{m}(f)$ being the coefficients of a continuous function?

The answer to this question is negative (as Theorem 2.2 will show). However, in view of the fact that $\left|\chi_{m}(t)\right| \leqq m^{1 / 2}$, we have at once

THEOREM 2.1. If $\left\{a_{m}\right\}$ is a sequence such that $\sum\left|a_{m}\right| m^{1 / 2}$ converges, then $\sum\left|a_{m} \chi_{m}(t)\right|$ converges uniformly on $[0,1]$.

REMARK 2.1. Theorem 2.1 does not appear to be very sharp in view of the result proved by P. L. Ul'janov [21, p. 45]: If $\left\{a_{m}\right\}$ is a sequence such that $\sum\left|a_{m}\right| m^{-1 / 2}$ converges, then $\sum\left|a_{m} \chi_{m}(t)\right|$ converges a.e. on $[0,1]$. However this condition is far from sufficient for the convergence of $\sum\left|a_{m} \chi_{m}(t)\right|$ everywhere on $[0,1]$. In fact, Ciesielski and Musielak noted [4, p. 64]: "The uniform convergence of $\sum\left|a_{m} \chi_{m}(t)\right|$ does not imply the convergence of $\sum\left|a_{m}\right|$ and conversely."

In order to show that Theorem 2.1 is sharp and to answer the question of Ciesielski and Musielak we require

LEMMA 2.1. If $\left\{\varepsilon_{m}\right\}$ is a sequence such that $\lim \varepsilon_{2^{m}}=0$, then there exists a bounded function $f$, constant on each interval of a collection whose union is $[0,1]$, such that

$$
\sum\left|a_{m}(f) m^{1 / 2} \varepsilon_{m}\right|<\infty
$$

and

$$
\sum\left|a_{m}(f) \chi_{m}(0)\right|=\infty \text {. }
$$

Proof. Choose a sequence $\left\{c_{m}\right\}$ such that $\sum c_{m}$ is conditionally convergent and $\sum\left|c_{m+1} \varepsilon_{2^{m}}\right|<\infty$. For example, if $\left|\varepsilon_{2^{m}}\right|<2^{-n}$ for $m \geqq N_{n}$, we may let $c_{m+1}=(-1)^{n} / n$ if $m+1=N_{n}$ and 0 elsewhere. 
Define $f(t)$ on $[0,1]$ as follows:

$$
\begin{aligned}
& f(t)=c_{0}+c_{1}+\cdots+c_{n-1}-c_{n} \text { on }\left(2^{-n}, 2^{-n+1}\right), \quad n=1,2, \ldots, \\
& =0 \quad \text { otherwise. }
\end{aligned}
$$

Then

$$
\begin{gathered}
\begin{aligned}
\int_{0}^{1} f(t) d t & =\lim _{n \rightarrow \infty}\left[\left(c_{0}-c_{1}\right) / 2+\left(c_{0}+c_{1}-c_{2}\right) / 4+\cdots+\left(c_{0}+\cdots+c_{n-1}-c_{n}\right) / 2^{n}\right] \\
= & \lim \left[\left(1-1 / 2^{n}\right) c_{0}-\left(c_{1}+c_{2}+\cdots+c_{n}\right) / 2^{n}\right]=c_{0},
\end{aligned} \\
\int_{0}^{1 / 2} f(t) d t-\int_{1 / 2}^{1} f(t) d t=-\left(c_{0}-c_{1}\right) / 2+\left(c_{0}+c_{1}-c_{2}\right) / 4+\cdots=c_{1}, \\
\int_{0}^{1 / 2^{n-1}} f(t) d t-\int_{1 / 2^{n-1}}^{1 / 2^{n}} f(t) d t \\
=-\left(c_{0}+\cdots+c_{n-1}-c_{n}\right) / 2^{n-1}+\cdots=c_{n+1} / 2^{n}, \quad n=1,2, \ldots
\end{gathered}
$$

Consequently

$$
\begin{gathered}
a_{0}(f)=\int_{0}^{1} f(t) d t=c_{0} \\
a_{1}(f)=\int_{0}^{1 / 2} f(t) d t-\int_{1 / 2}^{1} f(t) d t=c_{1}, \\
a_{2^{n}}(f)=2^{n / 2}\left[\int_{0}^{1 / 2^{n+1}} f(t) d t-\int_{1 / 2^{n+1}}^{1 / 2^{n}} f(t) d t\right]=c_{n+1} / 2^{n / 2} \quad(n=0,1, \ldots),
\end{gathered}
$$

and $a_{m}(f)=0$ otherwise.

Therefore

$$
\sum_{m=1}^{\infty}\left|a_{m}(f) m^{1 / 2} \varepsilon_{m}\right|=\sum_{m=0}^{\infty}\left|c_{m+1} \varepsilon_{2^{m}}\right|<\infty
$$

and

$$
\sum_{m=1}^{\infty}\left|a_{m}(f) \chi_{m}(0)\right|=\sum_{m=1}^{\infty}\left|c_{m}\right|=\infty
$$

which establishes our lemma.

REMARK 2.2. A similar argument would show that Lemma 2.1 remains valid if we replace $t=0$ in condition (2.4) by any $t_{0} \in[0,1]$.

We shall now prove

THEOREM 2.2. If $\varepsilon_{m} \downarrow 0$ and $\sum \varepsilon_{m} / m<\infty$, then there exists a continuous function $g$, linear on each interval of a collection whose union is $[0,1]$, such that

$$
\sum\left|a_{m}(g)\right| m^{1 / 2} \varepsilon_{m}<\infty
$$

and

$$
\sum\left|a_{m}(g) x_{m}(0)\right|=\infty .
$$

Proof. For clarity the proof will be divided into three parts. In part (i) we will show that the step function $f$ defined in Lemma 2.1 can be "connected" at the jumps 
producing a continuous function $h$ such that

$$
2^{n / 2}\left|a_{2^{n}}(f-h)\right| \leqq 2^{n} \int_{0}^{1 / 2^{n}}|f-h| \leqq K \cdot 2^{-n}, \quad n=1,2, \ldots,
$$

where $K$ is an arbitrary positive constant.

In part (ii) we construct a continuous function $g$ such that

$$
f(t) \leqq g(t) \leqq h(t)
$$

and

$$
\sum\left|a_{m}(f-g) m^{1 / 2} \varepsilon_{m}\right|<\infty, \quad m>0, \quad m \neq 2^{n}, \quad n=0,1, \ldots
$$

In part (iii) we show how the above relations imply that $g$ is our desired function. Part (i). Let $\left\{c_{m}\right\}$ and $f$ be as defined in Lemma 2.1 for the sequence $\left\{\varepsilon_{m}\right\}$ (actually $\sum c_{m}$ may be any conditionally convergent series). Assume (without loss of generality) that $\sum_{m=0}^{\infty} c_{m}=0$.

Define $h(t)$ on $\left[2^{-n}, 2^{-n+1}\right]$ for $n=1,2, \ldots$ as follows:

$$
\begin{array}{rlrl}
h(t) & =0, \quad t=2^{n}, 2^{-n+1}, \\
& =c_{0}+\cdots+c_{n-1}-c_{n}, \quad t \in I_{j} \equiv\left[2^{-n}+2^{-j_{n}}, 2^{-n+1}-2^{-j_{n}}\right] \\
& =\text { linear otherwise. } & \text { where } j_{n}>n \geqq 1,
\end{array}
$$

Now defining $h(0)=0$, we see that $h(t)$ is a continuous function.

We now choose the sequence $\left\{j_{n}\right\}$ so that $(2.8)$ is satisfied.

Letting $K$ be an arbitrary positive constant, we may choose $\left\{j_{n}^{1}\right\}_{n=1}^{\infty}$ such that $\int_{0}^{1}\left|h_{1}-f\right| \leqq K$, where $h_{1}$ is the function defined in (2.11) corresponding to $\left\{j_{n}^{1}\right\}$.

Choose $\left\{j_{n}^{2}\right\}_{n=2}^{\infty}$ such that $j_{n}^{2} \geqq j_{n}^{1}$ for $n=2,3, \ldots$ and

$$
2 \int_{0}^{1 / 2}\left|h_{2}-f\right| \leqq K / 2
$$

where $h_{2}$ is the function defined in (2.11) corresponding to $\left\{j_{n}^{2}\right\}$.

Clearly, we may define for $m=2,3,4, \ldots\left\{j_{n}^{m}\right\}_{n=m}^{\infty}$ and the corresponding $h_{m}$ such that $j_{n}^{m} \geqq j_{n}^{m-1}(n=m, m+1, \ldots)$ and

$$
2^{m-1} \int_{0}^{1 / 2^{m-1}}\left|h_{m}-f\right| \leqq K / 2^{m-1}
$$

Setting now $\left\{j_{n}^{n}\right\}=\left\{j_{n}\right\}$, the corresponding $h$ satisfies (2.8).

Part (ii). We now define $g$ by the formula (2.11), replacing $\left\{j_{n}\right\}$ by a sequence of integers $\left\{k_{n}\right\}$ such that $k_{n} \geqq j_{n}$, which implies (2.9), the sequence to be chosen such that $(2.10)$ is valid.

Setting now $E_{n}=\left\{m: \chi_{m}(t)=0\right.$ if $\left.t \notin\left[2^{-n}, 2^{-n+1}\right]\right\}$ for $n=1,2, \ldots$, we shall show

$$
\sum_{m \in E_{n}}\left|a_{m}(g) m^{1 / 2} \varepsilon_{m}\right|<K / 2^{n}
$$

for a suitable sequence $\left\{k_{n}\right\}$. 
Since $2^{n}+1=\min \left\{m: m \in E_{n}\right\}$, it follows that

$$
\begin{aligned}
a_{2\left(2^{n}+1\right)}(g) / 2^{(n+1) / 2} & =a_{2^{2}\left(2^{n}+1\right)}(g) / 2^{(n+2) / 2}=\cdots \\
& =a_{2^{k_{n}-n-1}\left(2^{n}+1\right)}(g) / 2^{\left(k_{n}-1\right) / 2} \\
& =-\left[c_{0}+c_{1} \cdots+c_{n-1}-c_{n}\right] / 2^{k_{n}+1}
\end{aligned}
$$

Therefore, by symmetry considerations

$$
\begin{aligned}
& \sum_{m \in \varepsilon_{n}, m<2^{k_{n}}}\left|a_{m}(g) m^{1 / 2} \varepsilon_{m}\right| \\
& \quad=\frac{2\left|c_{0}+\cdots+c_{n-1}-c_{n}\right|}{2^{k_{n}+1}}\left[2^{(n+1) / 2}\left(2^{n+1}+2\right)^{1 / 2} \varepsilon_{2\left(2^{n}+1\right)}+\cdots\right] \\
& \leqq 2^{3 / 2}\left|c_{0}+\cdots\right| \cdot\left[\left(\varepsilon_{2^{n+1}}\right) / 2^{k_{n}-n}+\left(\varepsilon_{2^{n+2}}\right) / 2^{k_{n}-n-1}+\cdots+\varepsilon_{2^{k n-1}}\right] .
\end{aligned}
$$

Letting now $m=2^{k_{n}-n}\left(2^{n}+1\right)$, we obtain

$$
\begin{aligned}
a_{m}(g) & =2^{\left(k_{n}\right) / 2}\left[\int_{2^{-n}}^{2-n+2-k_{n}-1} g(t) d t-\int_{2^{-n}+2^{-k_{n}-1}}^{2-n+2-k_{n}} g(t) d t\right] \\
& =-2^{\left(k_{n}\right) / 2}\left[c_{0}+\cdots+c_{n-1}-c_{n}\right] / 2^{k_{n}+2} \\
& =-\left[c_{0}+\cdots+c_{n-1}-c_{n}\right] / 2^{2} \cdot 2^{\left(k_{n}\right) / 2}, \\
a_{2 m}(g) & =a_{2 m+1}(g)=-\left[c_{0}+\cdots+c_{n-1}-c_{n}\right] / 2^{3} \cdot 2^{\left(k_{n}+1\right) / 2}, \\
a_{4 m}=a_{4 m+1} & =a_{4 m+2}=a_{4 m+3}=-\left[c_{0}+\cdots+c_{n-1}-c_{n}\right] / 2^{4} \cdot 2^{\left(k_{n}+2\right) / 2},
\end{aligned}
$$

Hence by symmetry considerations,

$$
\sum_{m \in E_{n}, m \geqq 2^{k}}\left|a_{m}(g) m^{1 / 2} \varepsilon_{m}\right| \leqq 2^{-1 / 2}\left|c_{0}+\cdots+c_{n-1}-c_{n}\right| \cdot\left[\varepsilon_{2^{k_{n}}}+\varepsilon_{2^{k_{n}+1}}+\cdots\right]
$$

Applying now the fact that $\sum \varepsilon_{2^{m}}$ converges (which is equivalent to $\sum \varepsilon_{m} / m$ converges) to (2.13) and (2.14), we may choose $k_{n}$ sufficiently large and obtain (2.12).

Therefore

$$
\begin{aligned}
\sum_{m>0 . m \neq 2^{n}, n=0.1 \ldots}\left|a_{m}(f-g) m^{1 / 2} \varepsilon_{m}\right| & =\sum_{m \in E_{n}, n=1,2 \ldots}\left|a_{m}(f-g) m^{1 / 2} \varepsilon_{m}\right| \\
& =\sum_{m \in E_{n}, n=1,2 \ldots}\left|a_{m}(g) m^{1 / 2} \varepsilon_{m}\right|<K
\end{aligned}
$$

which is exactly relation (2.10).

Part (iii). Relations (2.4), (2.8), and (2.9) imply

$$
\sum\left|a_{m}(g) \chi_{m}(0)\right|=\sum\left|a_{2^{n}}(g) \cdot 2^{n / 2}\right|=\infty .
$$


Relations (2.3), (2.8), (2.9), and (2.10) imply

$$
\sum\left|a_{m}(g) m^{1 / 2} \varepsilon_{m}\right|<\infty .
$$

This completes the proof.

COROllary 2.1. For every $\varepsilon>0$, there exists a continuous function $g$, differentiable except on a denumerable set, such that

$$
\sum\left|a_{m}(g)\right| m^{1 / 2-\varepsilon}<\infty \text { and } \sum\left|a_{m}(g) \chi_{m}(0)\right|=\infty .
$$

Proof. Let $\left\{\varepsilon_{m}\right\}=\left\{m^{-\varepsilon}\right\}$ in Theorem 2.2.

Also, since $\left|a_{m}(g)\right| \leqq 2^{1 / 2} \cdot M \cdot m^{-1 / 2}$ where $M=\max |g(t)|$, we obtain

COROllary 2.1'. For every $\varepsilon>0$, there exists a continuous function $g$, differentiable except on a denumerable set, such that

$$
\sum\left|a_{m}(g)\right|^{1+\varepsilon} m^{1 / 2}<\infty \text { and } \sum\left|a_{m}(g) \chi_{m}(0)\right|=\infty .
$$

REMARK 2.3. A similar argument would show that Theorem 2.2 remains valid if we replace $t=0$ in condition $(2.7)$ by any $t_{0} \in[0,1]$.

REMARK 2.4. It is known [5, p. 154] that if $g$ is continuous on $I=[0,1]$ and $\left|g^{\prime}(t)\right| \leqq M$ on $I-D$ where $D$ is a denumerable subset, then $g(t)$ satisfies a Lipschitz condition of order 1 . Also it is easily seen from Theorem $C[4$, p. 62] that if $g$ satisfies a Lipschitz condition of order $\alpha>0$, then $\sum\left|a_{m}(g) \chi_{m}(t)\right|$ converges uniformly on $[0,1]$. Thus for any function $g$ satisfying Theorem 2.2 we must have $\left|g^{\prime}(t)\right|$ unbounded on $I-D$.

REMARK 2.5. We do not know if the condition $\sum \varepsilon_{m} / m<\infty$ in Theorem 2.2 can be omitted.

REMARK 2.6. In Corollary 2.4 we shall prove that the function $g$ in Theorem 2.2 cannot be differentiable at the origin, and in Theorem 2.11 we will show that the function $g$ in Theorem 2.2 cannot be of bounded variation at the origin.

Letting $V$ denote the class of functions of bounded variation on $[0,1]$, we now state the following theorem due to P. L. Ul'janov [23, p. 374].

THEOREM D. If $f \in V$, then

$$
\sum\left|a_{m}(f)\right| m^{\beta}<\infty \text { for all } \beta<1 / 2 .
$$

For $\beta=1 / 2$ the theorem is false.

We note here that by modifying Ul'janov's proof slightly we obtain a stronger

THEOREM $\mathrm{D}^{\prime}$. (i) If $f \in V$, then

$$
\sum\left|a_{m}(f)\right| m^{1 / 2} \varepsilon_{m}<\infty
$$

for every sequence $\left\{\varepsilon_{m}\right\}, \varepsilon_{m} \downarrow 0$ such that $\sum \varepsilon_{2^{n}}<\infty$.

(ii) If $\varepsilon_{m} \downarrow 0$ and $\sum \varepsilon_{2^{n}}=\infty$, then there exists $f \in V$ such that

$$
\sum\left|a_{m}(f)\right| m^{1 / 2} \varepsilon_{m}=\infty .
$$


After we had solved the problem of Ciesielski and Musielak by Theorem 2.2, a paper appeared by Wang Si-Lei [18, p. 222] in which he proved the existence of an absolutely continuous function (and hence of bounded variation) whose HaarFourier series was not absolutely convergent at $t=2 / 3$. According to Theorem D with $\beta=0$, this produces a solution to the problem. We note here that in Theorem 2.2 we constructed a function of unbounded variation which was not absolutely convergent at $t=0$.

Wang's proof, although very interesting, relies upon a lemma of Bosanquet and Kestelman and is an existence proof only. Motivated by Wang's paper, we now apply the technique developed in the proof of Theorem 2.2 to actually exhibit such a function.

More precisely, we now prove (by construction)

THEOREM 2.3. There exists a monotone and absolutely continuous function $h$, differentiable except at $2 / 3$, such that

$$
\sum\left|a_{m}(h)\right| m^{1 / 2} \varepsilon_{m}<\infty \quad \text { for every } \varepsilon_{m} \downarrow 0 \text { satisfying } \sum \varepsilon_{2^{n}}<\infty
$$

and also

$$
\sum\left|a_{m}(h) \chi_{m}(2 / 3)\right|=\infty \text {. }
$$

Proof. According to Theorem $\mathrm{D}^{\prime}$, relation (2.15) is automatically satisfied for any absolutely continuous function. Hence we need only construct an absolutely continuous function of the given type which satisfies (2.16).

Now select a sequence $\left\{c_{m}\right\}$ such that $\sum c_{m}$ is conditionally convergent, and let $\left\{k_{n}\right\}$ be that sequence of integers satisfying

$$
\left(k_{n}\right) / 2^{n} \leqq 2 / 3<\left(k_{n}+1\right) / 2^{n}, \quad n=0,1, \ldots
$$

We now define an infinite sequence of disjoint intervals as follows:

$$
\begin{aligned}
E_{n} & =\left\{t:\left(k_{n}\right) / 2^{n} \leqq t<\left(k_{n}+1 / 2\right) / 2^{n}\right\}, & & n \text { even, } \\
& =\left\{t:\left(k_{n}+1 / 2\right) / 2^{n}<t \leqq\left(k_{n}+1\right) / 2^{n}\right\}, & & n \text { odd. }
\end{aligned}
$$

Notice that for $n=0,1, \ldots$ we have that $E_{2 n+2}$ is contiguous with and lies to the right of $E_{2 n}$, and $E_{2 n+3}$ is contiguous with and lies to the left of $E_{2 n+1}$.

Now define $f(t)$ on $[0,1]$ as follows:

$$
\begin{aligned}
f(t) & =c_{0}+\cdots+c_{n-1}-c_{n}, \quad t \in E_{n-1}, \quad n=1,2, \ldots, \\
& =\sum_{n=0}^{\infty} c_{n}, \quad t=2 / 3=\sum_{0}^{\infty} 2^{-2 n-1}
\end{aligned}
$$

Then, as in the proof of Lemma 2.1, we obtain

$$
\sum\left|a_{m}(f) \chi_{m}(2 / 3)\right|=\sum\left|c_{n}\right|=\infty .
$$


If we further assume that $\sum c_{n}$ is an alternating series with $\left|c_{n}\right| \downarrow 0$, we obtain that $f(t)$ is actually monotone as can easily be seen by comparing values on adjacent intervals.

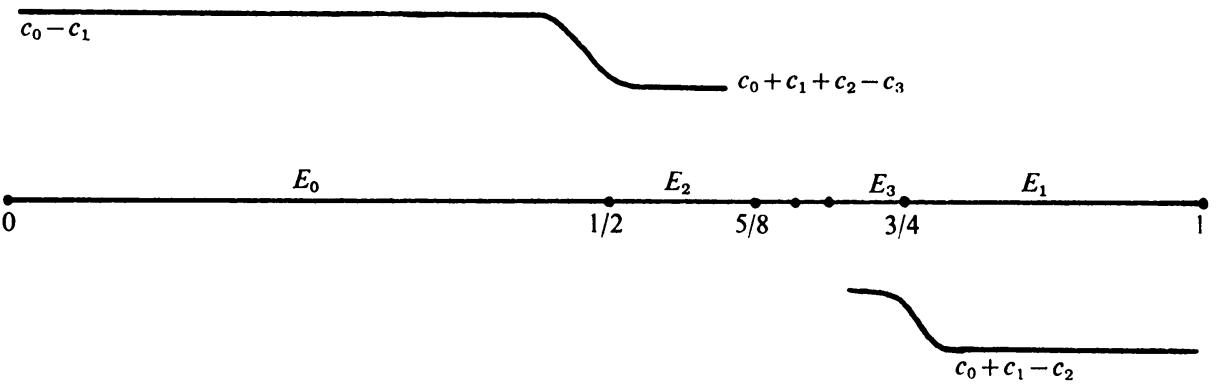

Figure 1

Analogous to part (i) of the proof of Theorem 2.2, we now "smooth" out our step function $f$ at the jumps (see Figure 1) to give us a continuous function $h$, differentiable except at $2 / 3$, satisfying (2.16), i.e. $\sum\left|a_{m}(h) \chi_{m}(2 / 3)\right|=\infty$.

Since $h(t)$ is monotone and absolutely continuous outside of every neighborhood of $t=2 / 3$ our result follows.

We note here that the choice of $2 / 3$ is not essential in Wang's argument and that his argument may be improved to include every dyadic irrational number. Thus we shall prove

THEOREM 2.4. For every dyadic irrational number $t_{0}$ there exists an absolutely continuous function $f$ such that $\sum\left|a_{m}(f) \chi_{m}\left(t_{0}\right)\right|=\infty$.

Proof. Following Wang we shall take advantage of the following result of L. S. Bosanquet and H. Kestelman [18, p. 222]:

Lemma A. Let $\left\{\phi_{n}(t)\right\}(n=1,2, \ldots)$ be a sequence of continuous functions on $[0,1]$. If for each $\psi \in L(0,1)$

$$
\sum_{n=1}^{\infty}\left|\int_{0}^{1} \psi(t) \phi_{n}(t) d t\right|<\infty,
$$

then $\sum_{n=1}^{\infty}\left|\phi_{n}(t)\right|$ is bounded on $[0,1]$.

We now let $t_{0}=\sum_{n=0}^{\infty} s_{n} 2^{-n}$ be a dyadic irrational (where $s_{n}=0$ or 1),

$$
\theta_{m}(t)=\int_{0}^{t} \chi_{m}(u) d u, \quad m=0,1, \ldots,
$$

and $k_{n}=k_{n}\left(t_{0}\right)$ a positive integer satisfying $\left(k_{n}\right) / 2^{n} \leqq t_{0}<\left(k_{n}+1\right) / 2^{n}$. Then for any absolutely continuous $f$,

$$
a_{m}(f)=\int_{0}^{1} f(t) \chi_{m}(t) d t=-\int_{0}^{1} f^{\prime}(t) \theta_{m}(t) d t .
$$


If for every absolutely continuous $f$, we have

$$
\begin{aligned}
\sum\left|a_{m}(f) \chi_{m}\left(t_{0}\right)\right| & =\sum\left|\int_{0}^{1} f^{\prime}(t) \theta_{m}(t) d t\right| \cdot\left|\chi_{m}\left(t_{0}\right)\right| \\
& =\sum_{n}\left|\int_{0}^{1} f^{\prime}(t) \theta_{2^{n}+k_{n}}(t) d t\right| 2^{n / 2}<\infty,
\end{aligned}
$$

then by Lemma $\mathrm{A}$ the series

$$
\sum\left|\theta_{2^{n}+k_{n}}(t)\right| \cdot 2^{n / 2}=\sum \theta_{2^{n}+k_{n}}(t) \cdot 2^{n / 2}
$$

must be bounded on $[0,1]$.

Choose now a subsequence $\left\{s_{n_{j}}\right\}$ of the sequence $\left\{s_{n}\right\}$ such that $s_{n_{j}+1}=0$ and $s_{n_{1}+2}=1, j=1,2, \ldots$ Then

$$
0<t_{0}-\sum_{n=0}^{n_{1}} s_{n} 2^{-n}=\sum_{n_{j}+1}^{\infty} s_{n} 2^{-n}=\sum_{n_{j}+2}^{\infty} s_{n} 2^{-n}<2^{-n_{j}-1} .
$$

Hence,

$$
\theta_{2^{n j}+k_{n j}}\left(t_{0}\right)=\left[t_{0}-\sum_{n=0}^{n_{j}} s_{n} 2^{-n}\right] 2^{\left(n_{j}\right) / 2}=2^{\left(n_{j}\right) / 2} \sum_{n_{j}+2}^{\infty} s_{n} 2^{-n}
$$

and so

$$
\sum_{j=1}^{\infty} 2^{\left(n_{j}\right) / 2} \theta_{2^{n j}+k_{n}}\left(t_{0}\right)=\sum_{j=1}^{\infty} 2^{n_{j}} \sum_{n=n_{j}+2}^{\infty} s_{n} 2^{-n} \geqq \sum_{j=1}^{\infty} 2^{n_{j}} \cdot 2^{-n_{j}-2}=\sum_{j=1}^{\infty} 1 / 4=\infty .
$$

Thus series (2.18) diverges at $t_{0}$.

REMARK 2.7. Theorem 2.4 is false for dyadic rationals. In Theorem 2.11 we will prove that the Haar-Fourier series of a function of bounded variation converges absolutely at all dyadic rationals.

It is easily shown $\left[23\right.$, p. 360] that if $|f(t)| \leqq M$ for all $t \in[0,1]$, then $\left|a_{m}(f)\right|$ $\leqq 2^{1 / 2} M \cdot m^{-1 / 2}$. Although the converse of this result is not true, we do have

THEOREM 2.5. (i) If $\left|a_{m}\right| \leqq K \varepsilon_{m} m^{-1 / 2}$, where $K$ is a constant and $\left\{\varepsilon_{m}\right\}$ is a quasimonotone sequence, i.e. $\left(\varepsilon_{m}\right) / m^{\beta} \downarrow 0$ for some $\beta$; such that $\sum \varepsilon_{2^{n}}<\infty$, then $\sum a_{m} \chi_{m}(t)$ is uniformly absolutely convergent and hence is the Haar-Fourier series of a bounded function.

(ii) If $\left\{\varepsilon_{m}\right\}$ is a quasi-monotone sequence such that $\sum \varepsilon_{2^{n}}=\infty$, then there exists a series $\sum a_{m} X_{m}(t)$ which is absolutely divergent everywhere and which is not the Fourier series of $a$ bounded function although $\left|a_{m}\right| \leqq K \varepsilon_{m} m^{-1 / 2}$.

Proof. (i) If $t_{0} \in[0,1]$, then from (2.20), which appears shortly, it follows that

$$
\sum_{m=1}^{\infty}\left|a_{m} \chi_{m}\left(t_{0}\right)\right| \leqq \sum_{n=1}^{\infty} K \varepsilon_{2^{n}}+k_{n} \leqq K^{\prime} \sum_{n=1}^{\infty} \varepsilon_{2^{n}}<\infty
$$

where $\left\{k_{n}\right\}$ is some sequence such that $0 \leqq k_{n}<2^{n}$. 
(ii) Setting $a_{m}=\left(\varepsilon_{2^{n+1}}\right) 2^{-n / 2}, 2^{n} \leqq m<2^{n+1}$, for $n=0,1, \ldots$, we obtain

$$
\sum_{m=1}^{\infty} a_{m} \chi_{m}(t)=\sum_{n=1}^{\infty} \varepsilon_{2^{n+1}} r_{n}(t) \quad \text { on }(0,1)
$$

where $\left\{r_{m}(t)\right\}$ are the Rademacher functions [1, p. 51]. But then $\sum a_{m} \chi_{m}(t)$ is absolutely divergent everywhere and the Rademacher series above does not represent an essentially bounded function since $\sum \varepsilon_{2^{n}}$ diverges [9, p. 455]. But since the Haar-Fourier series of an integrable function $f$ converges a.e. to $f$ (Theorem A), we must have that the Haar-Fourier series of a bounded function is essentially bounded.

The relation on the numbers $\left|a_{m}\right|$ follows from (2.20).

REMARK 2.8. If $\left|a_{m}\right| \leqq K \varepsilon_{m} m^{-1 / 2}$ where $\left\{\varepsilon_{m}\right\}$ is quasi-monotone and $\sum\left(\varepsilon_{2^{n}}\right)^{2}<\infty$, then $\sum a_{m} \chi_{m}(t)$ is a Haar-Fourier series since, by $(2.21)$, we then have $\sum\left(a_{m}\right)^{2}<\infty$.

REMARK 2.9. The inequality $\left|a_{m}\right| \leqq K \varepsilon_{m} m^{-3 / 2}$ for $\varepsilon_{m} \downarrow 0, \sum \varepsilon_{2^{n}}=\infty$ does not imply that $\sum a_{m} \chi_{m}(t)$ is the Haar-Fourier series of a function of bounded variation. In fact, setting

$$
a_{m}=(-1)^{n} \varepsilon_{2^{n}} 2^{-3 n / 2} \text { for } 2^{n} \leqq m<2^{n+1}, \quad n=0,1, \ldots,
$$

we obtain that

$$
\sum_{m=1}^{\infty} a_{m} \chi_{m}(t)=\sum_{n=1}^{\infty}(-1)^{n} \varepsilon_{2^{n}} 2^{-n} r_{n}(t) \text { on }(0,1) .
$$

But, by a result of A. I. Rubinstein [17, p. 143], the function represented by series (2.19) is not of class $\operatorname{Lip}(1,1)$, i.e., is not equivalent to a function of bounded variation.

P. L. Ul'janov has proved [23, p. 378], [21, p. 45]; see also [22, p. 439].

THEOREM E. If $a_{m} \downarrow 0$, then for $\sum\left|a_{m} \chi_{m}(t)\right|$ to be uniformly convergent on $[0,1]$ it is both necessary and sufficient that one of the following conditions be satisfied:

(a) $\sum a_{m} m^{-1 / 2}<\infty$,

(b) $\sum\left|a_{m} \chi_{m}(t)\right|$ converges for at least one point $t_{0} \in[0,1]$,

(c) $\sum a_{m} \chi_{m}(t)$ is the Fourier series of a bounded function $f$.

In order to generalize this result to quasi-monotone coefficients we shall prove the following (cf. [19, p. 203] where an equivalent definition of quasi-monotonic is used).

LEMMA 2.2. If $\left\{b_{m}\right\}$ is quasi-monotonic, then

$$
\begin{gathered}
b_{2^{m}+k_{m}} \leqq K b_{2^{m}} \leqq K^{2} b_{2^{m-1}+k_{m-1}} \\
\sum_{n=m+1}^{\infty}\left(b_{2^{n}+k_{n}}\right) 2^{n} \leqq K \sum_{n=2^{m}}^{\infty} b_{n} \leqq K^{2} \sum_{n=m-1}^{\infty}\left(b_{2^{n}+k_{n}}\right) 2^{n}
\end{gathered}
$$


for $m=1,2, \ldots$ where $K$ is a positive constant and $\left\{k_{n}\right\}$ is any sequence of integers such that $0 \leqq k_{n}<2^{n}$.

\section{Proof.}

$$
\begin{aligned}
\sum_{n=m+1}^{\infty}\left[\left(b_{2^{n}+k_{n}}\right) /\left(2^{n}+k_{n}\right)^{\beta}\right] 2^{n}\left(2^{n}+k_{n}\right)^{\beta} & \\
\leqq & \sum_{n=m+1}^{\infty}\left[\sum_{i=2^{n-1}}^{2^{n-1}}\left(b_{i}\right) / i^{\beta}\right] 2\left(2^{n+1}\right)^{\beta} \leqq 2^{2 \beta+1} \sum_{i=2^{m}}^{\infty} b_{i} .
\end{aligned}
$$

Relation (2.20) and the other half of inequality (2.21) are proved similarly.

We shall now apply (2.21) with $b_{m}=a_{m} m^{-1 / 2}$ and prove

THEOREM 2.6. In Theorem $\mathrm{E}$ we may replace the condition $\left\{a_{m}\right\}$ is monotonic by the condition $\left\{a_{m}\right\}$ is quasi-monotonic.

Proof. Assuming $t_{0} \in[0,1]$ and $\sum a_{m} m^{-1 / 2}<\infty$ we obtain

$$
\sum_{m=2^{n}}^{\infty}\left|a_{m} \chi_{m}\left(t_{0}\right)\right| \leqq \sum_{m=n}^{\infty}\left(a_{2^{m}+k_{m}}\right) 2^{m / 2} \leqq K \sum_{m=2^{n-1}}^{\infty} a_{m} m^{-1 / 2} \rightarrow 0
$$

as $n \rightarrow \infty$, where $0 \leqq k_{n}=k_{n}\left(t_{0}\right)<2^{n}$ and $K$ is a positive constant.

We now show that condition (b) implies condition (a). Assuming

$$
\sum_{m=1}^{\infty}\left|a_{m} \chi_{m}\left(t_{0}\right)\right|=D<\infty
$$

we obtain

$$
D=\sum_{m=1}^{\infty}\left|a_{m} \chi_{m}\left(t_{0}\right)\right| \geqq \sum_{n=N}^{\infty}\left(a_{2^{n}+k_{n}}\right) 2^{n / 2} \geqq K \sum_{n=2^{N+1}}^{\infty} a_{n} n^{-1 / 2}
$$

where $0 \leqq k_{n}<2^{n}, N=N\left(t_{0}\right)$, and $K$ is a positive constant.

Assuming now condition (c) we have that

$$
\sup _{n}\left|\sum_{0}^{n} a_{m}(f) \chi_{m}(0)\right|=\sup _{n} \sum_{0}^{n}\left|a_{m}(f) \chi_{m}(0)\right|<\infty
$$

and hence condition (b) is satisfied. This completes our proof.

In Theorem 2.2 we showed that $\sum\left|a_{m}(f)\right|<\infty$ does not imply $\sum\left|a_{m}(f) \chi_{m}(t)\right|<\infty$ for every $t \in[0,1]$, for a continuous $f$. Hence the question naturally arises as to whether $\sum\left|a_{m}(f) \chi_{m}(t)\right|<\infty$ on $[0,1]$ implies that $\sum\left|a_{m}(f)\right|<\infty$ for continuous or differentiable $f$ ? The answer is no and we now prove

THEOREM 2.7. There exists a differentiable function $f(t)$ on $[0,1]$ such that $\sum\left|a_{m}(f) \chi_{m}(t)\right|<\infty$ on $[0,1]$ and $\sum\left|a_{m}(f)\right|^{2-\varepsilon}=\infty$ for every $\varepsilon>0$.

Proof. A. M. Olevskii has shown [24, p. 4] that for every complete orthonormal system there exists a differentiable function $f$ such that $\sum\left|a_{m}(f)\right|^{2-\varepsilon}=\infty$ for every 
$\varepsilon>0$. The fact that the Haar-Fourier series of $f$ converges absolutely on $[0,1]$ follows immediately from Corollary 2.4 , which follows presently.

Applying now the fact that $\left|a_{m}(f)\right| \leqq 2^{1 / 2} M m^{-1 / 2}$, where $M=\max |f(t)|$, we obtain

COROLlary 2.2. There exists a differentiable function $f$ on $[0,1]$ such that $\sum\left|a_{m}(f) \chi_{m}(t)\right|<\infty$ on $[0,1]$ and for every $\varepsilon>0$

$$
\sum\left|a_{m}\right|^{1-\varepsilon} m^{-1 / 2}=\sum\left|a_{m}\right| m^{-1 / 2} m^{\varepsilon}=\infty .
$$

REMARK 2.10. Olevskir has shown [21, p. 44] that if $\sum\left|a_{m}\right|^{2-\varepsilon}<\infty$ for some $\varepsilon$ satisfying $0<\varepsilon<2$, then $\sum\left|a_{m} \chi_{m}(t)\right|$ converges a.e. on $[0,1]$.

REMARK 2.11. By utilizing the fact $[7$, p. $620 ; 8$, p. 1281] that there exists a function $f \in \operatorname{Lip}(1 / 2)$ such that $\sum\left|a_{m}(f)\right|$ diverges, it follows immediately from Theorem $\mathrm{C}$ that there exists a continuous function $f$ such that $\sum\left|a_{m}(f) \chi_{m}(t)\right|$ converges uniformly on $[0,1]$ and yet $\sum\left|a_{m}(f)\right|=\infty$.

It may be of interest to note that we have a theorem analogous to Theorem 2.2 for the trigonometric system. Thus we shall now prove

THEOREM 2.8. For every null sequence $\left\{\varepsilon_{k}\right\}$ there exists a continuous function $f$ such that

(a) $\sum\left[a_{k}(f) \cos k t+b_{k}(f) \sin k t\right]$ converges to $f(t)$ uniformly,

(b) $\sum\left[a_{k}(f) \cos k t+b_{k}(f) \sin k t\right]$ is nowhere absolutely convergent,

(c) $\sum\left[\left|a_{k}(f)\right|+\left|b_{k}(f)\right|\right] \varepsilon_{k}<\infty$, where $a_{k}(f), b_{n}(f)$ denote the trigonometric Fourier coefficients of $f$.

Proof. Let $\left\{N_{m}\right\}$ be an increasing sequence of integers such that $\left|\varepsilon_{k}\right|<2^{-m}$ if $N_{m} \leqq k<N_{m+1}$. Then setting

$$
a_{k}=\left(N_{m+1}-N_{m}\right)^{-1} \quad \text { if } N_{m} \leqq k<N_{m+1}
$$

we obtain for a fixed $\varepsilon>0$

$$
\begin{aligned}
\sum_{k=N_{1}}^{\infty} a_{k}^{2}(\log k)^{1+\varepsilon} & =\sum_{N_{1}}^{N_{2}-1}+\sum_{N_{2}}^{N_{3}-1}+\cdots \\
& \leqq \frac{\left(\log N_{2}\right)^{1+\varepsilon}}{N_{2}-N_{1}}+\frac{\left(\log N_{3}\right)^{1+\varepsilon}}{N_{3}-N_{2}}+\cdots \\
& \leqq \frac{2\left(\log N_{2}\right)^{1+\varepsilon}}{N_{2}}+\frac{2\left(\log N_{3}\right)^{1+\varepsilon}}{N_{3}}+\cdots<\infty
\end{aligned}
$$

for a suitable sequence $\left\{N_{m}\right\}$, chosen such that $\left|a_{k}\right| \downarrow 0$.

But Payley and Zygmund have shown [29, p. 219] that since series (2.22) is convergent, we then have $\sum \pm a_{k} \cos k t$ uniformly convergent for some alternation of signs. Also, since $\left|a_{k}\right| \downarrow 0$ and $\sum\left|a_{k}\right|=\infty$, it follows [29, p. 232] that $\sum\left|a_{k} \cos k t\right|$ diverges everywhere on $[0,2 \pi]$.

Condition (c) is satisfied by construction. 
For the Haar functions the principle of localization holds, i.e. if two functions coincide in some interval, then their Fourier series converge or diverge simultaneously at any interior point. With respect to absolute convergence the principle remains true for the Haar functions although it fails for the trigonometric system [3, II, p. 187].

In view of this it seems appropriate to consider further absolute convergence at specific points. We shall need the following definitions in which $f(t)=0$ if $t \notin[0,1]$ :

$$
\begin{gathered}
\omega\left(f, t_{0}, h\right)=\sup _{\left|t-t_{0}\right| \leqq h}\left|f(t)-f\left(t_{0}\right)\right| ; \\
\omega_{1}\left(f, t_{0}, h\right)=\int_{t_{0}-h}^{t_{0}+h}\left|f(t)-f\left(t_{0}\right)\right| d t ; \\
f \in \operatorname{Lip}\left(\alpha, t_{0}\right) \text { if }\left|f(t)-f\left(t_{0}\right)\right| \leqq k\left|t-t_{0}\right|^{\alpha},
\end{gathered}
$$

where $0<\alpha \leqq 1$, in a neighborhood of $t_{0}$ ( $K$ is a positive constant).

We now prove (cf. Theorem $\mathrm{C}$ )

TheOREM 2.9. If $\sum_{n=1}^{\infty} 2^{n} \omega_{1}\left(f, t_{0}, 2^{-n}\right)<\infty$ for some $t_{0} \in[0,1]$, then

$$
\sum\left|a_{m}(f) \chi_{m}\left(t_{0}\right)\right|<\infty \text {. }
$$

Proof. Choose integers $k_{n}\left(t_{0}\right)$ and $k_{n}^{\prime}\left(t_{0}\right)$ such that $\left(k_{n}\right) / 2^{n}<t_{0} \leqq\left(k_{n}+1\right) / 2^{n}$ and $\left(k_{n}^{\prime}\right) / 2^{n} \leqq t_{0}<\left(k_{n}^{\prime}+1\right) / 2^{n}$. Then

$$
\begin{aligned}
& \sum\left|a_{m}(f) \chi_{m}\left(t_{0}\right)\right| \leqq \sum\left[\left|a_{2^{n}+k_{n}}(f)\right|+\left|a_{2^{n}+k_{n}^{\prime}}(f)\right|\right] 2^{n / 2} \\
& =\sum_{n=1}^{\infty} 2^{n}\left\{\left|\int_{\left(k_{n}\right) / 2^{n}}^{\left(k_{n}+1 / 2\right) / 2^{n}}\left[f(t)-f\left(t+2^{-n-1}\right)\right] d t\right|\right. \\
& \left.+\left|\int_{\left(k_{n}^{\prime}\right) / 2}^{\left(k_{n}^{\prime}+1 / 2\right) / 2^{n}}\left[f(t)-f\left(t+2^{-n-1}\right)\right] d t\right|\right\} \\
& \leqq 4 \sum_{n=1}^{\infty} 2^{n} \omega_{1}\left(f, t_{0}, 2^{-n}\right)
\end{aligned}
$$

We have immediately the following

Corollary 2.3. If $\sum \omega\left(f, t_{0}, 2^{-n}\right)<\infty$ for some $t_{0} \in[0,1]$, then

$$
\sum\left|a_{m}(f) \chi_{m}\left(t_{0}\right)\right|<\infty \text {. }
$$

By a slight modification of the proof of Theorem 2.9 we also obtain

THEOREM 2.9'. If $f \in \operatorname{Lip}\left(\alpha, t_{0}\right)$ for some $\alpha>0$ and $t_{0} \in[0,1]$, then

$$
\sum\left|m^{\alpha-\varepsilon} a_{m}(f) \chi_{m}\left(t_{0}\right)\right|
$$

converges for every $\varepsilon>0$.

COROLlary 2.4. If has a right and left hand derivative at $t_{0}$, then $\sum\left|a_{m}(f) \chi_{m}\left(t_{0}\right)\right|$ converges. 
Remark 2.12. Theorem $2.9^{\prime}$ is false for $\alpha=1, \varepsilon=0$ since if $f(t)=(1-2 t)$ we obtain $a_{m}(f)=2^{-1-3 n / 2}$ if $2^{n} \leqq m<2^{n+1}$ and consequently $\sum\left|m a_{m}(f) \chi_{m}(t)\right|$ diverges on $[0,1]$.

Corollary 2.3 is best possible in the sense of

THEOREM 2.10. For every positive null sequence $\left\{\varepsilon_{n}\right\}$ there exists a continuous function $f$ such that

$$
\sum \omega\left(f, 0,2^{-n}\right) \cdot \varepsilon_{n}<\infty
$$

and

$$
\sum\left|a_{m}(f) \chi_{m}(0)\right|=\infty .
$$

Proof. Choose a sequence $\left\{c_{m}\right\}$ satisfying:

(i) $\sum c_{n}$ is an alternating conditionally convergent series,

(ii) $\left|c_{n}\right| \leqq\left|c_{n-1}\right|$,

(iii) $\sum\left|c_{n} \varepsilon_{n}\right|<\infty$.

For example, we may let $c_{m}=(-1)^{m} / n\left(N_{n}-N_{n-1}\right)$ for $N_{n-1} \leqq m<N_{n}$, where $\left\{N_{n}\right\}$ is chosen such that $\left|c_{n}\right| \downarrow 0$ and $\left|\varepsilon_{m}\right|<2^{-n}$ for $m \geqq N_{n-1}$.

Define $f(t)$ on $\left[2^{-n}, 2^{-n+1}\right]$ for $n=1,2, \ldots$ as follows:

$$
\begin{aligned}
f(t) & =0, \quad t=2^{-n}, 3 \cdot 2^{-n-1}, 2^{-n+1}, \\
& =c_{n}, \quad t=7 \cdot 2^{-n-2} \\
& =\text { linear and continuous on the contiguous intervals. }
\end{aligned}
$$

Defining now $f(0)=0$, we obtain from (ii) that $\omega\left(f, 0,2^{-n}\right) \leqq\left|c_{n}\right|$ if $n \geqq 1$ which together with (iii) implies (2.23). Also

$$
\left|a_{2^{n}}(f)\right|=2^{n / 2}\left|c_{n+1} 2^{-n-3}-\left(c_{n+2} 2^{-n-4}+c_{n+3} 2^{-n-5}+\cdots\right)\right|,
$$

and hence

$$
2^{n / 2}\left|a_{2^{n}}(f)\right|=2^{-3}\left|c_{n+1}-\left(c_{n+2} 2^{-1}+c_{n+3} 2^{-2}+\cdots\right)\right| \geqq 2^{-3}\left|c_{n+1}\right|
$$

for $n=1,2, \ldots$ by (i) and (ii) which implies (2.24).

REMARK 2.13. A similar proof would suffice for any dyadic rational.

Since a function $f$ of bounded variation has right and left hand limits everywhere, it follows from Theorem 1.3 that the Haar-Fourier series of $f$ converges at all dyadic rationals (cf. [23, p. 368, Theorem 3]). We will strengthen this result and prove

THEOREM 2.11. If $t_{0}$ is a dyadic rational and a point of bounded variation for $f(t)$, then $\sum\left|a_{m}(f) \chi_{m}\left(t_{0}\right)\right|<\infty$.

Proof. We shall prove the theorem for $t_{0}=0$. A similar argument will suffice for any dyadic rational. 
Assume that

$$
\sum\left|a_{m}(f) \chi_{m}(0)\right|=\sum 2^{n}\left|\int_{0}^{1 / 2^{n+1}} f(t) d t-\int_{1 / 2^{n+1}}^{1 / 2^{n}} f(t) d t\right| \equiv \sum\left|c_{n}\right|=\infty .
$$

Then

$$
\begin{aligned}
2 c_{n-1}-c_{n} & =2^{n}\left[\int_{0}^{1 / 2^{n}}-\int_{1 / 2^{n}}^{1 / 2^{n-1}}\right]-2^{n}\left[\int_{0}^{1 / 2^{n+1}}-\int_{1 / 2^{n+1}}^{1 / 2^{n}}\right] \\
& =2^{n+1} \int_{1 / 2^{n+1}}^{1 / 2^{n}} f(t) d t-2^{n} \int_{1 / 2^{n}}^{1 / 2^{n-1}} f(t) d t
\end{aligned}
$$

and thus

$$
\left|2 c_{n-1}-c_{n}\right| \leqq V\left(f ;\left[2^{-n-1}, 2^{-n+1}\right]\right) \equiv V\left(2^{-n-1}, 2^{-n+1}\right),
$$

the variation of $f$ on $\left[2^{-n-1}, 2^{-n+1}\right]$.

Further

$$
\begin{aligned}
2 V(0,1) & =V(1 / 4,1)+[V(1 / 4,1)+V(1 / 8,1 / 4)]+[V(1 / 8,1 / 4)+V(1 / 16,1 / 8)]+\cdots \\
& \geqq V(1 / 4,1)+V(1 / 8,1 / 2)+V(1 / 16,1 / 4)+\cdots \\
& \geqq \sum_{n=1}^{\infty}\left|2 c_{n-1}-c_{n}\right| \geqq \sum_{n=1}^{\infty}\left(\left|2 c_{n-1}\right|-\left|c_{n}\right|\right)=\sum\left|c_{n}\right|=\infty .
\end{aligned}
$$

But this is a contradiction if $f$ is of bounded variation on [0, 1]. By an obvious adoption of this proof we see that we need only require that $f$ is of bounded variation in a neighborhood of 0 .

REMARK 2.14. In the proof of Theorem 2.11 we did not actually need the hypothesis that 0 was a point of bounded variation for $f(t)$; we only used the fact that if

$$
F(t)=2^{n} \int_{1 / 2^{n}}^{1 / 2^{n-1}} f(x) d x \text { for } t \in\left(2^{-n}, 2^{-n+1}\right), \quad n=1,2, \ldots,
$$

then 0 was a point of bounded variation for $F(t)$.

REMARK 2.15. $\sum\left|a_{m}(f) \chi_{m}(0)\right|<\infty$ does not imply that $f(t)$ is of bounded variation at the origin.

REMARK 2.16. It is known (see [1, p. 256]; [23, p. 368]) that if $f(t)$, a function of bounded variation, has a discontinuity at a dyadic irrational, then its HaarFourier series diverges there.

REMARK 2.17. Since a function of bounded variation is differentiable a.e., we have by Corollary 2.4 and Theorem 2.11 that $\sum\left|a_{m}(f) \chi_{m}(t)\right|$ converges except possibly on a set of measure zero of dyadic irrationals.

We now show that the converse of Corollary 2.3 is false. More precisely, we now prove

THEOREM 2.12. If $f$ is continuous on $[0,1]$, then $\sum\left|a_{m}(f) \chi_{m}(t)\right|<\infty$ on $[0,1]$ does not imply $\sum \omega\left(f, 0,2^{-n}\right)<\infty$. 
Proof. Let $\omega\left(2^{-n}\right) \downarrow 0$ arbitrarily slowly as $n \rightarrow \infty$. Set

$$
\begin{aligned}
f(t) & =\omega\left(2^{-n}\right), \quad t=2^{-n} \text { for } n=0,1, \ldots, \\
& =0, \quad t=0, \\
& =\text { linear and continuous on }\left[2^{-n-1}, 2^{-n}\right] .
\end{aligned}
$$

Then $f(t)$ is monotone and is differentiable at all dyadic irrationals. Hence by Theorem 2.11 and Corollary 2.4 we obtain that $\sum\left|a_{m}(f) \chi_{m}(t)\right|$ converges for every $t \in[0,1]$.

Since $\omega\left(f, 0,2^{-n}\right)=\omega\left(2^{-n}\right)$, this completes our proof.

We now investigate the sharpness of Theorem $C$ and prove

THEOREM 2.13. If $\omega(\delta) \downarrow 0, \omega(\delta) / \delta \uparrow$ as $\delta \downarrow 0$ and $\sum \omega\left(n^{-1}\right) n^{-1}=\infty$, then there exists a continuous function $f$ such that

$$
\text { (a) } \omega(\delta, f)=O[\omega(\delta)] ; \quad \text { (b) } \sum\left|a_{m}(f) \chi_{m}(0)\right|=\infty .
$$

Proof. Setting $c_{n}=(-1)^{m} \omega\left(2^{-n}\right)$ for $n \geqq 1$ we obtain

(i) $\sum c_{n}$ is an alternating conditionally convergent series,

(ii) $\left|c_{n}\right| \leqq\left|c_{n-1}\right|$,

(iii) $2\left|c_{n}\right| \geqq\left|c_{n-1}\right|$.

Now defining $f(t)$ as in Theorem 2.10, we obtain

$$
\begin{aligned}
\omega\left(f, 2^{-n}\right) & \leqq 2 \max \left(\left|c_{n-2}\right|,\left|c_{n-3}\right| 2^{-1}, \ldots,\left|c_{1}\right| 2^{-n+3}\right) \\
& \leqq 2\left|c_{n-2}\right| \leqq 8\left|c_{n}\right|=8 \omega\left(2^{-n}\right)
\end{aligned}
$$

if $n \geqq 3$ by (ii) and (iii), and

$$
2^{n / 2}\left|a_{2^{n}}(f)\right| \geqq\left|c_{n+1}\right| 2^{-3} \quad \text { by (i) and (ii). }
$$

Consequently

$$
\sum\left|a_{m}(f) \chi_{m}(0)\right|=\sum\left|a_{2^{n}}(f)\right| 2^{n / 2} \geqq 2^{-3} \sum\left|c_{n}\right|=\infty .
$$

REMARK 2.18. Ul'janov has independently proved Theorem 2.13 [26, p. 982]; $[28$, p. 200].

REMARK 2.19. Theorem 2.13 is similar to a result of B. I. Golubov for the HaarFourier coefficients of a continuous function [8, p. 1277].

Letting

$$
E_{n}(f)=\inf _{\left\{a_{m}\right\}} \sup _{t}\left|f(t)-\sum_{m=1}^{n} a_{m} \chi_{m}(t)\right|,
$$

where the infimum is extended over all real sequences $\left\{a_{m}\right\}$, we shall prove the following:

Corollary 2.5. If $f$ is continuous, then $\sum E_{n}(f) n^{-1}<\infty$ implies the uniform convergence of $\sum\left|a_{m}(f) \chi_{m}(t)\right|$ on $[0,1]$. 
COROLLARY 2.6. If $\left\{E_{n}\right\}$ is a sequence such that $E_{n} \downarrow 0, n E_{n} \uparrow$, and $\sum E_{n} n^{-1}=\infty$, then there exists a continuous $f$ such that
(a) $E_{n}(f)=O\left(E_{n}\right)$,
(b) $\sum\left|a_{m}(f) \chi_{m}(0)\right|=\infty$.

Proof of Corollary 2.5. By a lemma of Golubov [8, p. 1274] we have that if $f(t)$ is continuous on $[0,1]$, then

$$
E_{n}(f) \leqq \omega\left(n^{-1}, f\right) \leqq 6 E_{n}(f), \quad n \geqq 2 .
$$

Hence our desired result follows immediately from Theorem $\mathrm{C}$.

Proof of Corollary 2.6. Let $\omega\left(n^{-1}\right)=E_{n}$ in Theorem 2.13. Then (b) fo'lows from Theorem 2.13 and (a) follows from inequality (2.25) and Theorem 2.13.

RemarK 2.20. Corollaries 2.5 and 2.6 are similar to theorems of S. N. Bernstein for the trigonometric system [3, II, pp. 154, 165] and to theorems of B. I. Golubov for convergence of the Haar-Fourier coefficients [7, p. 621]; [8, p. 1281].

3. Functions represented by Haar and Walsh series. The relationships between the coefficients of Haar (and Walsh) series and the functions which the series represent have been much investigated (see [8], [10], [11], [18] and [23]). This section is concerned with these relationships.

L. A. Balasov has shown [2, p. 631] that a necessary and sufficient condition that $f(t)=\sum a_{m} r_{m}(t)$ have a derivative at at least one point is that the limit of $a_{m} 2^{m}$ exist. We shall prove

THEOREM 3.1. If $\lim a_{m} m^{3 / 2}=0$, then $f(t)=\sum a_{m} \chi_{m}(t)$ has a derivative on a dense set.

Proof. Let $t_{0}=.1010 \ldots$, where the expansion is given in binary form, and let $\left\{t_{k}\right\}$ be a sequence of numbers such that $t_{k} \neq t_{0}, \lim t_{k}=t_{0}$. Assuming that the first $p$ places of $t_{k}$ are the same as $t_{0}$ and the $(p+1)$ th place differs, we have that $p \rightarrow \infty$ as $k \rightarrow \infty$. Then (cf. [2, p. 632]) $\left|t_{k}-t_{0}\right| \geqq 2^{-p-3}$ and

$$
\begin{aligned}
\lim _{t_{k} \rightarrow t_{0}}\left|\frac{f\left(t_{k}\right)-f\left(t_{0}\right)}{t_{k}-t_{0}}\right| & =\lim _{t_{k} \rightarrow t_{0}}\left|\frac{\sum_{m=2^{p-2}}^{\infty} a_{m}\left[\chi_{m}\left(t_{k}\right)-\chi_{m}\left(t_{0}\right)\right]}{t_{k}-t_{0}}\right| \\
& \leqq \lim _{p \rightarrow \infty}\left|\frac{\varepsilon_{p} \sum_{n=p-2}^{\infty} 2^{-3 n / 2} \cdot 2^{n / 2}}{2^{-p-3}}\right|
\end{aligned}
$$

where $\varepsilon_{p} \rightarrow 0$ as $p \rightarrow \infty$, and this last expression equals

$$
\lim _{p \rightarrow \infty}\left|16 \cdot \varepsilon_{p}\right|=0
$$

Hence $f^{\prime}(t)$ exists at $t_{0}=.1010 \ldots$

In order to complete the proof we need only notice that this argument is actually valid for $t_{0}$ plus any dyadic rational number. 
By similar reasoning applied to $\left\{w_{m}(t)\right\}$, the Walsh orthonormal system [1, p. 59], we obtain the following

THEOREM 3.2. If $\lim a_{m} m^{2}=0$, then $f(t)=\sum a_{m} w_{m}(t)$ has a derivative on a dense set.

In order to obtain our next results we need the following lemma which follows from Minkowski's inequality:

LEMMA 3.1. If $V_{p}\left(f_{m}\right)$ denotes the pth variation of $f_{m}(t)$, then

(i) if $0<p \leqq 1$,

$$
V_{p}^{p}\left(\sum_{n=1}^{\infty} f_{m}\right) \leqq \sum_{m=1}^{\infty} V_{p}^{p}\left(f_{m}\right)
$$

(ii) if $p \geqq 1$,

$$
V_{p}\left(\sum_{m=1}^{\infty} f_{m}\right) \leqq \sum_{m=1}^{\infty} V_{p}\left(f_{m}\right)
$$

We now state two theorems which are immediate consequences of Lem ma 3.1.

THEOREM 3.3. (i) If $0<p \leqq 1$, then $\sum\left|a_{m}\right|^{p} m<\infty$ implies $f(t)=\sum a_{m} w_{m}(t)$ is of bounded pth variation.

(ii) If $p \geqq 1$, then $\sum\left|a_{m}\right| m^{1 / p}<\infty$ implies $f(t)=\sum a_{m} w_{m}(t)$ is of bounded pth variation.

THEOREM 3.4. (i) If $0<p \leqq 1$, then $\sum\left|a_{m}\right|^{p} m^{p / 2}<\infty$ implies $f(t)=\sum a_{m} \chi_{m}(t)$ is of bounded pth variation.

(ii) If $p \geqq 1$, then $\sum\left|a_{m}\right| m^{1 / 2}<\infty$ implies $f(t)=\sum a_{m} \chi_{m}(t)$ is of bounded pth variation.

REMARK 3.1. By applying Theorem (4.1) in [12] we obtain:

(i) if $\lim \varepsilon_{m}=0$, then $\sum\left|a_{m} m \varepsilon_{m}\right|<\infty$ does not imply $f(t)=\sum a_{m} w_{m}(t)$ is differentiable a.e.

(ii) if $\lim \varepsilon_{m}=0$, then $\sum\left|a_{m} m^{1 / 2} \varepsilon_{m}\right|<\infty$ does not imply $f(t)=\sum a_{m} \chi_{m}(t)$ is differentiable a.e.

REMARK 3.2. Part (i) of Theorems 3.3 and 3.4 are best possible by part (iii) of Theorem (3.1) in [12].

Remark 3.3. Part (ii) of Theorem 3.4 is best possible in the sense that if lim $\varepsilon_{m}$ $=0$, then $\sum\left|a_{m}\right| m^{1 / 2} \varepsilon_{m}<\infty$ does not imply $f(t)=\sum a_{m} \chi_{m}(t)$ is bounded. This is easily seen by setting $a_{2^{n}}=2^{-n / 2}$ at selected $n$ 's and $a_{m}=0$ otherwise.

REMARK 3.4. V. A. Matveev has proved [11, p. 1405] that if $f \in L$ and $\sum\left|a_{m}(f)\right| m^{1 / 2}<\infty$, then $f(t)$ is equivalent to a function of bounded variation. However, since $f(t)=\sum a_{m}(f) \chi_{m}(t)$ a.e. whenever $f \in L$, this result follows immediately from Theorem 3.4 for $p=1$.

B. I. Golubov has shown ([8, p. 1296]; see also Wang [18, p. 224]) that if $f$ is continuous and $a_{m}(f)=o\left(m^{-3 / 2}\right)$ with respect to the Haar system, then $f$ is a constant. We shall prove, using a simpler method, 
THEOREM 3.5. If $f(t)=\sum a_{m} \chi_{m}(t)$ has the Darboux property and $a_{m}=o\left(m^{-3 / 2}\right)$, then $f$ is a constant.

Proof. Assume $\left|a_{m}\right| \leqq\left(m^{-3 / 2}\right) \varepsilon / 4$ if $m \geqq 2^{N}$. Then $x, y \in\left((i-1) / 2^{N}, i / 2^{N}\right), i=1$, $2, \ldots, 2^{N}$ implies

$$
|f(x)-f(y)| \leqq(\varepsilon / 2) \sum_{m=0}^{\infty} 2^{-N-m}=\varepsilon / 2^{N} .
$$

Hence, letting $x \rightarrow(i-1) / 2^{N}, y \rightarrow i / 2^{N}$ in inequality (4.1) we obtain by the Darooux property

$$
|f(x)-f(y)| \leqq \varepsilon / 2^{N} \quad \text { if } x, y \in\left[(i-1) / 2^{N}, i / 2^{N}\right] .
$$

But this implies that

$$
|f(x)-f(y)| \leqq \varepsilon \quad \text { if } x, y \in[0,1],
$$

which completes the demonstration.

By utilizing Theorem 1.1 and the fact that an approximately continuous function has the Darboux property (or just by noticing that the proof of Theorem 3.5 goes through easily for approximately continuous functions) we obtain

COROLlaRY 3.1. If $f$ is bounded and approximately continuous and $a_{m}(f)$ $=o\left(m^{-3 / 2}\right)$, then $f$ is a constant .

Golubov has further proved ([8, p. 1295]; see also Wang [18, p. 226] and Matveev [10, p. 108]) that if $f$ is continuous on $[0,1], 0<\alpha \leqq 1$, and $a_{m}(f)=$ $O\left(m^{-1 / 2-\alpha}\right)$ with respect to the Haar system, then $f \in \operatorname{Lip} \alpha$. We will prove a somewhat more general result by a simpler method.

THEOREM 3.6. If $f(t)=\sum a_{m} \chi_{m}(t)$ has the Darboux property and $a_{m}=O\left(m^{-1 / 2-\alpha}\right)$, then $f \in \operatorname{Lip} \alpha$.

Proof. Analogous to the proof of Theorem 3.5 we obtain

$$
|f(x)-f(y)| \leqq K^{\prime}\left(2^{-n \alpha}+2^{-(n+1) \alpha}+\cdots\right)=K / 2^{n \alpha}
$$

whenever $x, y \in\left[i / 2^{n},(i+1) / 2^{n}\right]$.

Consequently, if $2^{-n-1}<|x-y| \leqq 2^{-n}$, then

$$
|f(x)-f(y)| \leqq 2 K \cdot 2^{-n \alpha}<4 K|x-y|^{\alpha},
$$

which produces our desired result.

Applying Theorem 1.1 we immediately obtain

COROLLARY 3.2. If $f$ is bounded and approximately continuous and $a_{m}(f)=$ $O\left(m^{-1 / 2-\alpha}\right)$, then $f \in \operatorname{Lip} \alpha$.

4. Applications of Haar series to general orthonormal series. The system of Haar functions is an easily defined example of a complete system of bounded orthonormal functions which is not uniformly bounded. Hence, when examining 
any conjecture about general orthonormal series, it is often advantageous to consider the special case of the Haar system. In many cases such consideration will also show that certain results are best possible for unbounded complete orthonormal systems. For example, if $\left\{\phi_{m}\right\}$ is a sequence of functions with variations $\left\{V_{m}\right\}$, then $\sum\left|a_{m}\right| V_{m}<\infty$ implies that $f(t)=\sum a_{m} \phi_{m}(t)$ is a function of bounded variation; and as Remark 3.2 shows, the theorem is best possible for the Haar system. We now proceed to some other results.

For $\left\{\phi_{m}\right\}$ orthonormal on $[a, b]$, we have that if $\sum\left|a_{m}\right|$ converges, then $\sum\left|a_{n} \phi_{m}(t)\right|$ converges a.e. there $[1$, p. 63$]$. We now state

THEOREM 4.1. (i) If $\sup _{a \leqq t \leqq b}\left|\phi_{m}(t)\right|=M_{m}$, then $\sum\left|a_{m}\right| M_{m}<\infty$ implies that $\sum\left|a_{m} \phi_{m}(t)\right|<\infty$ on $[a, b]$.

(ii) For unbounded complete orthonormal systems the condition in (i) may not be relaxed even though the series may be a uniformly convergent Fourier series.

Proof. Part (i) is obvious.

Part (ii) follows by combining the fact that $M_{m}=O\left(m^{1 / 2}\right)$ for the Haar system with Lemma 2.1.

For uniformly bounded orthonormal systems we have that the Fourier coefficients of any integrable function tend to zero $[3, I$, p. 66]. We now prove

THEOREM 4.2. (i) If $\left\{\phi_{m}\right\}$ is orthonormal on $[a, b]$ and $\sup _{a \leqq t \leqq b}\left|\phi_{m}(t)\right|=M_{m}$, then

$$
\int_{a}^{b} f(t) \phi_{m}(t) d t=o\left(M_{m}\right) \quad \text { for every } f \in L .
$$

(ii) For unbounded complete orthonormal systems (4.1) may not in general be improved.

Proof. (i) Let $M=M_{m}$ in the proof given in Bary's book [3, I, p. 66].

(ii) Combine the fact that $M_{m}=O\left(\mathrm{~m}^{1 / 2}\right)$ for the Haar system with the result of Ul'janov [23, p. 363] that $a_{m}(f)=o\left(m^{1 / 2}\right)$ cannot be improved.

For a uniformly bounded orthonormal system $\left\{\phi_{m}\right\}$ on $[a, b]$, we have that the relation $a_{m} \rightarrow 0$ is a necessary condition for the convergence a.e. of the orthogonal series $\sum a_{m} \phi_{m}(t)[1$, p. 7]. We shall prove

THEOREM 4.3. (i) If $\sup \left|\phi_{m}(t)\right|=M_{m}$ and $\sum a_{m} \phi_{m}(t)$ is uniformly convergent, then $a_{m} \cdot M_{m}=o(1)$.

(ii) For unbounded complete orthonormal systems (i) may not in general be improved even though the series may be a uniformly convergent Fourier series of a continuous function.

Proof. Part (i) is immediate.

(ii) Use the result of Ul'janov [23, p. 365] that for continuous $f$, the relation $a_{m}(f) \cdot m^{1 / 2}=o(1)$ cannot be improved for the Haar system.

REMARK 4.1. The uniformity condition in part (i) may not be omitted [1, p. 8]. 
We now prove

THEOREM 4.4. (i) If $\left\{\phi_{m}(t)\right\}$ is a normal system (i.e. $\int_{a}^{b} \phi_{m}^{2}(t) d t=1, m=0,1, \ldots$ ) and $\sup _{a \leqq t \leqq b}\left|\phi_{m}(t)\right|=M_{m}$, then $\sum\left|a_{m}\right| / M_{m}$ converges whenever $\sum a_{m} \phi_{m}(t)$ is uniformly absolutely convergent.

(ii) For unbounded complete orthonormal systems the conclusion in (i) may not in general be strengthened.

Proof.

$$
\begin{aligned}
\sum_{m}^{n}\left|a_{i}\right| / M_{i} & =\sum_{m}^{n}\left(\left|a_{i}\right| / M_{i}\right) \int_{a}^{b} \phi_{i}^{2}(t) d t \\
& \leqq \sum_{m}^{n}\left|a_{i}\right| \int_{a}^{b}\left|\phi_{i}(t)\right| d t=\int_{a}^{b}\left(\sum_{m}^{n}\left|a_{i} \phi_{i}(t)\right|\right) d t .
\end{aligned}
$$

(ii) If $\delta_{n} \uparrow \infty$ arbitrarily slowly, choose a nonnegative sequence $\left\{\varepsilon_{m}\right\}$ such that

$$
\sum \varepsilon_{n}<\infty \text { and } \sum \varepsilon_{n} \delta_{2^{n}}=\infty \text {. }
$$

For example, if $\left\{n_{k}\right\}$ is chosen such that $\delta_{2^{n k}} \geqq 2^{k}$, then let $\varepsilon_{n_{k}}=2^{-k}, k=1,2, \ldots$, and $\varepsilon_{m}=0$ otherwise.

Setting now $a_{m}=2^{-n / 2} \varepsilon_{n}$ for $2^{n} \leqq m<2^{n+1}$, we obtain that $\sum\left|a_{m} \chi_{m}(t)\right| \leqq \sum \varepsilon_{m}$ and

$$
\sum\left(\left|a_{m}\right| / m^{1 / 2}\right) \delta_{m} \geqq 2^{-1 / 2} \sum \varepsilon_{n} \delta_{2^{n}}=\infty .
$$

REMARK 4.2. In part (i) the uniformity condition may not be omitted for the Haar system because by choosing $\left\{a_{m}\right\}$ such that $a_{m}=0$ if $m \neq 2^{n}+1(n=0,1, \ldots)$ we obtain that $\sum a_{m} \chi_{m}(t)$ is absolutely convergent on $[0,1]$.

REMARK 4.3. In part (i) the condition of normality may not be omitted. To see this we consider the orthogonal system $\left\{\phi_{n}(t)\right\}$ where $\phi_{n}(t)=n^{1 / 2}$ on $E_{n}$ and 0 elsewhere, where $\left\{E_{n}\right\}$ is a mutually disjoint infinite sequence of subsets of $[a, b]$. Then $\sum\left|a_{m} \phi_{m}(t)\right|$ is uniformly convergent whenever $a_{m} m^{1 / 2} \rightarrow 0$. However, this condition does not imply that $\sum a_{m} m^{-1 / 2}$ converges.

Of course, if $M_{m} \rightarrow 0$, then the fact that $\sum a_{m} \phi_{m}(t)$ is uniformly absolutely convergent does not imply that $\sum\left|a_{m}\right| / M_{m}$ converges.

W. Orlicz has proved [16, p. 232]

Theorem G. Let $d_{n} \geqq 0(n=1,2, \ldots)$ and $\sum d_{n}^{2}=\infty$. If $\left\{\phi_{n}(t)\right\}$ is a uniformly bounded orthonormal system on $[0,1]$, then there exists a continuous function $f$ for which the Fourier coefficients $a_{n}$ with respect to the system $\left\{\phi_{m}(t)\right\}$ satisfy the condition

$$
\sum d_{n}\left|a_{n}\right|=\infty
$$

A. M. Olevskii has noted $[15$, p. 654$]$ that the conclusion of Orlicz's theorem is not valid for the Haar system. We notice, however, that the conclusion is still valid for the Haar system if $\left\{d_{n}\right\}$ is quasi-monotonic. This follows easily since by Lemma 
2.2 we then have $\sum d_{m}^{2} \chi_{m}^{2}(t)=\infty$ a.e. and our desired conclusion is obtained by a remark of Orlicz [16, p. 233].

\section{REFERENCES}

1. G. Alexits, Convergence problems of orthogonal series, Pergamon Press, New York, 1961.

2. L. A. Balasov, On gap series, Izv. Akad. Nauk SSSR Ser. Mat. 29 (1965), 631-644. (Russian)

3. N. Bari, A treatise on trigonometric series. I, II, Pergamon Press, New York, 1961.

4. Z. Ciesielski and J. Musielak, On absolute convergence of Haar series, Colloq. Math. 7 (1959), 61-65.

5. J. Dieudonné, Foundations of modern analysis, Academic Press, New York, 1960.

6. G. Faber, Uber die Orthogonalfunktionen des Herrn Haar, Jber. Deutsch. Math.-Verein. 19 (1910), 104-112.

7. B. I. Golubov, Fourier series of continuous functions in the Haar system, Dokl. Akad. Nauk SSSR 156 (1964), 247-250=Soviet Math. Dokl. 5 (1964), 620-623.

8. - Fourier series of continuous functions with respect to the Haar system, Izv. Akad. Nauk SSSR Ser. Mat. 28 (1964), 1271-1296. (Russian)

9. F. R. Keogh, On Rademacher series with bounded sums, J. London Math. Soc. 33 (1958), 454-455.

10. V. A. Matveev, On Fourier-Haar coefficients, Izv. Vyš̌. Učebn. Zaved. Matematika 1965, no. 6(49), 103-112. (Russian)

11. - On variations of functions and on Fourier coefficients with respect to the Haar and Schauder systems, Izv. Akad. Nauk SSSR Ser. Mat. 30 (1966), 1397-1419. (Russian)

12. J. R. McLaughlin, Functions represented by Rademacher series, Pacific J. Math. 27 (1968).

13. M. E. Munroe, Introduction to measure and integration, Addison-Wesley, Reading, Mass., 1953.

14. A. M. Olevskiĭ, Divergent Fourier series for continuous functions, Dokl. Akad. Nauk SSSR 138 (1961), 545-548 = Soviet Math. Dokl. 2 (1961), 1382-1386.

15. - The divergence of orthogonal series and the Fourier coefficients of continuous functions with respect to complete systems, Sibirsk, Mat. Ż. 4 (1963), 647-656. (Russian)

16. W. Orlicz, Theorie der Orthogonalwicklungen. III, Bull. Acad. Polon. Sci. Sér. Sci. A. 1932, 229-238.

17. A. I. Rubenstein, On gap series, Izv. Vysš. Učebn. Zaved. Matematika 1963, no. 3(34), 137-148. (Russian)

18. Wang Sei-Lei, On Haar series, Acta Math. Sinica 16 (1966), 211-222=Chinese Math. Acta 8 (1966), 222-233.

19. O. Szász, Quasi-monotone series, Amer. J. Math. 70 (1948), 203-206.

20. V. A. Skvorcov, A theorem of Cantor type for the Haar system, Vestnik Moskov. Univ. Ser. I Mat. Meh. 1964, no. 5, 3-6. (Russian)

21. P. L. Ul'janov, Divergent Fourier series, Uspehi Mat. Nauk 16 (1961), no. 3(99), 61-142 $=$ Russian Math. Surveys 16 (1961), no. 3, 1-75.

22. - Haar series, Dokl. Akad. Nauk SSSR 148 (1963), 532-534= Soviet Math. Dokl. 4 (1963), 437-440.

23. —_, On Haar series, Mat. Sb. 63 (105) (1964), 356-391. (Russian)

24. - Solved and unsolved problems in the theory of trigonometric and orthogonal series, Uspehi Mat. Nauk 19 (1964), no. 1(115), 3-69= Russian Math. Surveys 19 (1964), no. 1, 1-62. 
25. P. L. Ul'janov, Haar series, Vestnik Moskov. Univ. Ser. I Mat. Meh. 1965, no. 4, 35-43. (Russian)

26. - On absolute and unconditional convergence, Dokl. Akad. Nauk SSSR 169 (1966), 536-538 = Soviet Math. Dokl. 7 (1966), 981-983.

27. —- On some properties of Haar series, Mat. Zametki 1 (1967), no. 1, 17-24. (Russian)

28. - On absolute and uniform convergence of Fourier series, Mat. Sb. 72 (114) (1967), 193-225 = Math. USSR Sbornik 1 (1967).

29. A. Zygmund, Trigonometric series. I, Cambridge Univ. Press, New York, 1959.

Pennsylvania State University,

University Park, Pennsylvania. 